\title{
MULTIPLICITY OF POLYNOMIALS ON TRAJECTORIES OF POLYNOMIAL VECTOR FIELDS IN $\mathrm{C}^{3}$
}

\author{
ANDREI GABRIELOV \\ Department of Mathematics, Purdue University \\ W. Lafayette, IN 47907-1395 \\ E-mail: agabriel@math.purdue.edu \\ FRÉDÉRIC JEAN \\ École Nationale Supérieure de Techniques Avancées \\ 32, Bd Victor, F-75739 Paris cedex 15 \\ E-mail:fjean@ensta.fr \\ JEAN-JACQUES RISLER \\ Équipe Analyse Alqébrique, Institut de Mathématiques, Université Paris 6 \\ Case 82, 2 place Jussieu, F-75252 Paris Cedex 05 \\ E-mail: risler@math.jussieu.fr
}

\begin{abstract}
Let $\xi$ be a polynomial vector field on $\mathbf{C}^{n}$ with coefficients of degree $d$ and $P$ be a polynomial of degree $p$. We are interested in bounding the multiplicity of a zero of a restriction of $P$ to a non-singular trajectory of $\xi$, when $P$ does not vanish identically on this trajectory. Bounds doubly exponential in terms of $n$ are already known $([9,5,10])$. In this paper, we prove that, when $n=3$, there is a bound of the form $p+2 p(p+d-1)^{2}$. In Control Theory, such a bound can be used to give an estimate of the degree of nonholonomy for a system of polynomial vector fields (this degree expresses the level of Lie-bracketing needed to generate the tangent space at each point).
\end{abstract}

1. Introduction. The problem of bounding the order of a polynomial along the trajectory of a polynomial vector field comes from at least two different theories: Transcendental Number Theory $([9])$, and Control Theory $([5,10])$. Let us state it in a more precise way:

Let $\xi(x)$ be a vector field on $\mathbf{R}^{n}$ or $\mathbf{C}^{n}$, with polynomial coordinates of degree not greater than $d$, and such that $\xi(O) \neq 0, P$ be a polynomial of degree $p, \gamma$ the integral

1991 Mathematics Subject Classification: 32S70, 93B25, 93B05.

Received by the editors: January 24, 1997; in the revised form: March 13, 1998.

The paper is in final form and no version of it will be published elsewhere. 
curve of $\xi$ by $O$. Assuming that $P_{\mid \gamma} \not \equiv 0$, the problem is to bound the order of the power series $P_{\mid \gamma}$ in terms of $p, d, n$.

A bound of the form $2^{n^{2} 2^{n}} d^{n^{2} 2^{n}} p^{n}$ has been obtained by Nesterenko ([9]). Independently, Gabrielov ([5]) obtained a bound of the form $d^{2^{n-2}-1} p^{2 n-1}(d+p)^{2^{n-2}}$. These two bounds are doubly exponential in terms of the dimension $n$, and probably far from being optimal. In particular, all the examples we know are simply exponential in term of $n$.

Actually, since the writing of this paper, Gabrielov ([7]) found a bound simply exponential in $n$ and polynomial in $d$ and $p$, namely

$$
2^{2 n-1} \sum_{k=1}^{n}[p+(k-1)(d-1)]^{2 n}
$$

In the plane case $(n=2)$, a better bound of the form $p(p+d-1)+1$ is known $([4,10])$.

In this paper, we prove that when $n=3$, there is also a better bound, of the form $p+2 p(p+d-1)^{2}$. The proof uses the technique of estimation of multiplicities of Pfaffian intersection developed in [6].

The content of the paper is as follows:

Section 2 gives facts from [6] about the multiplicity of deformation of an analytic germ at $O \in \mathbf{C}^{n}$ defined by equations $\phi_{1}=\ldots=\phi_{k}=0$. This is meaningful, even if the above intersection is not isolated. However, in this paper we need only the isolated case, which is simpler that the general one, as explained in Section 2.

Section 3 contains the proof of the estimation, and an application to the degree of nonholonomy is postponed to Section 4.

\section{Multiplicities and deformations}

2.1. Multiplicity. Let $\phi_{1}(x), \ldots, \phi_{n}(x)$ be germs of analytic functions in $\left(\mathbf{C}^{n}, O\right)$. We set $Z\left(\phi_{1}, \ldots, \phi_{n}\right)$ for the analytic germ at $O$ defined by the intersection $\left\{\phi_{1}=\ldots\right.$ $\left.\ldots=\phi_{n}=0\right\}$. When this intersection is isolated, one has $\operatorname{dim}_{\mathbf{C}} \frac{\mathbf{C}\left\{X_{1}, \ldots, X_{n}\right\}}{\left(\phi_{1}, \ldots, \phi_{n}\right)}<+\infty$ and its multiplicity is defined as

$$
e\left(\phi_{1}, \ldots, \phi_{n}\right)=\operatorname{dim}_{\mathbf{C}} \frac{\mathbf{C}\left\{X_{1}, \ldots, X_{n}\right\}}{\left(\phi_{1}, \ldots, \phi_{n}\right)} .
$$

Recall also that when $\phi_{1}(x), \ldots, \phi_{n}(x)$ are polynomials of degrees $q_{1}, \ldots, q_{n}$, we have $e\left(\phi_{1}, \ldots, \phi_{n}\right) \leq q_{1} \ldots q_{n}$ by Bézout's theorem.

R e mark 1. This definition of multiplicity can be used also in the following case (see [3], p. 137). Let $I$ be an ideal in the ring of the analytic functions in $\left(\mathbf{C}^{n}, O\right)$ such that the local ring $\mathbf{C}\left\{X_{1}, \ldots, X_{n}\right\} / I$ is of dimension 1 and Cohen-Macaulay. For an analytic function $\phi$, when the intersection $\{I=0\} \cap\{\phi=0\}$ is isolated, its multiplicity is

$$
e(\phi, I)=\operatorname{dim}_{\mathbf{C}} \frac{\mathbf{C}\left\{X_{1}, \ldots, X_{n}\right\}}{(\phi, I)} .
$$

This formula implies indeed (1): the ideal $I=\left(\phi_{1}, \ldots, \phi_{n-1}\right)$ is a complete intersection and so the ring $\mathbf{C}\left\{X_{1}, \ldots, X_{n}\right\} /\left(\phi_{1}, \ldots, \phi_{n-1}\right)$ is Cohen-Macaulay. We will use also this formula when $I$ is the ideal of a reduced curve. In this case $\mathbf{C}\left\{X_{1}, \ldots, X_{n}\right\} / I$ is a reduced 1-dimensional ring and therefore Cohen-Macaulay ([8], p. 187). 
Let us consider an ideal $I=\left(\phi_{1}, \ldots, \phi_{n-1}\right)$ such that $\Gamma$ defined as $\left\{\phi_{1}=\ldots\right.$ $\left.\ldots=\phi_{n-1}=0\right\}$ is a 1-dimensional analytic germ in $\mathbf{C}^{n} \cdot \Gamma=\bigcup \Gamma_{j}$ is the decomposition of $\Gamma$ into irreducible components and, for all $j$, we denote by $I_{j}$ the prime ideal associated to the component $\Gamma_{j}$. Let $\phi$ be an analytic function such that $Z(\phi, I)$ is an isolated intersection. Then, from Remark 1 , the multiplicity $e\left(\phi, I_{j}\right)$ is defined for all $j$. If we denote by $\nu_{j}$ the multiplicity of the component $\Gamma_{j}$ in $\Gamma$, we have (see [11], p. 84, 92)

$$
e(\phi, I)=\sum_{j} \nu_{j} e\left(\phi, I_{j}\right)
$$

Let $\tau=\varphi / \eta$ be a meromorphic function on $\Gamma_{j}$ which is not identically zero. We can extend $\varphi$ and $\eta$ to $\hat{\varphi}$ and $\hat{\eta}$, germs of analytic functions in $\left(\mathbf{C}^{n}, O\right)$. The intersections $\Gamma_{j} \cap\{\hat{\varphi}=0\}$ and $\Gamma_{j} \cap\{\hat{\eta}=0\}$ are isolated and we define the multiplicity $e\left(\tau, I_{j}\right)=e\left(\hat{\varphi}, I_{j}\right)-e\left(\hat{\eta}, I_{j}\right)$. Notice that this multiplicity does not depend on the extensions $\hat{\varphi}$ and $\hat{\eta}$ but only on the functions on the curve $\varphi$ and $\eta$.

If $\alpha$ and $\beta$ are two 1-forms, the ratio $\left.\alpha\right|_{\Gamma_{j}} /\left.\beta\right|_{\Gamma_{j}}$ is a meromorphic function on $\Gamma_{j}$. Thus the multiplicities $e\left(\left.\alpha\right|_{\Gamma_{j}} /\left.\beta\right|_{\Gamma_{j}}, I_{j}\right)$ can be defined as above. Following formula (2), we denote the sum of these multiplicities by

$$
e\left(\frac{\left.\alpha\right|_{\Gamma}}{\left.\beta\right|_{\Gamma}}, I\right)=\sum_{j} \nu_{j} e\left(\frac{\left.\alpha\right|_{\Gamma_{j}}}{\left.\beta\right|_{\Gamma_{j}}}, I_{j}\right) .
$$

2.2. Multiplicity of deformation. If $\phi(x)$ is a germ of an analytic function in $\left(\mathbf{C}^{n}, O\right)$ we call deformation a germ $\widetilde{\phi}(x, \epsilon)$ of an analytic function in $\left(\mathbf{C}^{n+1}, O\right)$ such that $\widetilde{\phi}(x, 0)=$ $\phi(x)$. We denote by $\phi^{\epsilon}(x)$ the function $\widetilde{\phi}(x, \epsilon)$ for $\epsilon$ fixed.

Let $\widetilde{\phi_{1}}(x, \epsilon), \ldots, \widetilde{\phi_{n}}(x, \epsilon)$ be deformations of the analytic functions $\phi_{1}(x), \ldots, \phi_{n}(x)$. We define the multiplicity of deformation $\#\left(\widetilde{\phi_{1}}, \ldots, \widetilde{\phi_{n}}\right)$ of the ideal $\left(\widetilde{\phi_{1}}, \ldots, \widetilde{\phi_{n}}\right)$ as the number of isolated zeroes of $Z\left(\phi_{1}^{\epsilon}, \ldots, \phi_{n}^{\epsilon}\right), \epsilon \neq 0$, counted with their multiplicities, converging to the origin $O$ when $\epsilon \rightarrow 0$.

Remark 2. If the functions $\phi_{1}^{\epsilon}(x), \ldots, \phi_{n}^{\epsilon}(x)$ are polynomial in $x$ of degrees $q_{1}, \ldots, q_{n}$ independent of $\epsilon$ and if the sets $Z\left(\phi_{1}^{\epsilon}, \ldots, \phi_{n}^{\epsilon}\right)$, for small $\epsilon \neq 0$, are of dimension 0, then Bézout's theorem can be applied to each one of these sets and we obtain $\#\left(\widetilde{\phi_{1}}, \ldots, \widetilde{\phi_{n}}\right) \leq q_{1} \cdots q_{n}$.

We will use the multiplicity of deformation in another case (definition introduced in [6]). Let $\bar{\Gamma}$ be a reduced 2-dimensional analytic subspace of $\mathbf{C}^{n+1}$ such that $\bar{\Gamma} \cap\{\epsilon=0\}$ is a curve without embedded components. Such a space $\bar{\Gamma}$ is called a deformation of $\bar{\Gamma}^{0}=\bar{\Gamma} \cap\{\epsilon=0\}$. We denote by $\bar{\Gamma}^{\epsilon}$ the intersection $\bar{\Gamma} \cap\{\epsilon=$ const. $\}$ and by $\bar{I}$ the ideal associated to $\bar{\Gamma}$. Note that the spaces $\bar{\Gamma}^{\epsilon}$ are reduced, for small $\epsilon \neq 0$, while $\bar{\Gamma}^{0}$ is not necessarily reduced.

Let $\widetilde{\phi}(x, \epsilon)$ be a deformation of an analytic function $\phi$. The multiplicity of the deformation, $\downarrow(\widetilde{\phi}, \bar{I})$, is defined as the number of isolated zeroes, counted with their multiplicities, of $\left.\widetilde{\phi}\right|_{\Gamma^{\epsilon}}, \epsilon \neq 0$, converging to the origin $O$ when $\epsilon \rightarrow 0$. Notice that, for this definition, the deformation $\widetilde{\phi}(x, \epsilon)$ need not be defined on the whole space but only on $\bar{\Gamma}$.

Re mark 3 . The multiplicity $\#\left(\widetilde{\phi_{1}}, \ldots, \widetilde{\phi_{n}}\right)$ depends in general on the deformations $\widetilde{\phi}_{i}(x, \epsilon)$. However if the intersection $Z\left(\phi_{1}, \ldots, \phi_{n}\right)$ is isolated, this multiplicity depends only on the germs $\phi_{1}(x), \ldots, \phi_{n}(x)$ and we have $\#\left(\widetilde{\phi_{1}}, \ldots, \widetilde{\phi_{n}}\right)=e\left(\phi_{1}, \ldots, \phi_{n}\right)$ (see for 
instance [1], p. 76). The same is true for the multiplicity $\downarrow(\widetilde{\phi}, \bar{I})$. If the zeroes of $\left.\widetilde{\phi}\right|_{\bar{\Gamma}^{0}}$ are isolated, then we have $\downarrow(\widetilde{\phi}, \bar{I})=e\left(\phi, \bar{I}^{0}\right)\left(\bar{I}^{0}\right.$ is the ideal associated to $\left.\bar{\Gamma}^{0}\right)$.

We would like to have a formula like (2) for the multiplicities of deformation. Such a formula would rely the two definitions of multiplicity of deformation. For that, we need to consider only a restricted class of deformations. Let us specify what are these restrictions.

Let $\widetilde{\phi_{1}}(x, \epsilon), \ldots, \widetilde{\phi_{n-1}}(x, \epsilon)$ and $\widetilde{\phi}(x, \epsilon)$ be deformations of the $n$ analytic functions $\phi_{1}, \ldots, \phi_{n-1}$ and $\phi$. We say that the deformation $\left(\widetilde{\phi}, \widetilde{\phi_{1}}, \ldots, \widetilde{\phi_{n-1}}\right)$ is special (or that $(\widetilde{\phi}, \widetilde{I})$ is special, with $\left.\widetilde{I}=\left(\widetilde{\phi_{1}}, \ldots, \widetilde{\phi_{n-1}}\right)\right)$ if the following conditions are satisfied:

- the ideal $I=\left(\phi_{1}, \ldots, \phi_{n-1}\right)$ defines a 1-dimensional analytic subspace $\Gamma$ in $\mathbf{C}^{n}$,

- the intersections $Z\left(\phi^{\epsilon}, \phi_{1}^{\epsilon}, \ldots, \phi_{n-1}^{\epsilon}\right)$, for small $\epsilon \neq 0$, are isolated.

In particular, if $Z(\phi, I)$ is isolated, any deformation $(\widetilde{\phi}, \widetilde{I})$ is special.

Let us consider now a special deformation $(\widetilde{\phi}, \widetilde{I})$. The multiplicity $\#(\widetilde{\phi}, \widetilde{I})$ is defined and we denote by $\Gamma$ the 1-dimensional analytic subspace defined by the ideal $I$. The set $\widetilde{\Gamma}$ defined as $\left\{\widetilde{\phi_{1}}=\ldots=\widetilde{\phi_{n-1}}=0\right\}$ is a 2 -dimensional analytic subspace in $\mathbf{C}^{n+1}$. Let $\widetilde{\Gamma}=\bigcup \bar{\Gamma}_{j}$ be the decomposition of $\widetilde{\Gamma}$ into irreducible components and, for all $j, \bar{I}_{j}$ be the prime ideal associated to the component $\bar{\Gamma}_{j}$ and $\mu_{j}$ be the multiplicity of the component $\bar{\Gamma}_{j}$ in $\widetilde{\Gamma}$ (see [11]). Each component $\bar{\Gamma}_{j}$ is a reduced 2-dimensional analytic subspace in $\mathbf{C}^{n+1}$ and the intersection $\bar{\Gamma}_{j} \cap\{\epsilon=0\}$ is a curve without embedded components. Hence the multiplicities $\sharp\left(\widetilde{\phi}, \bar{I}_{j}\right)$ are defined.

Since the spaces $\bar{\Gamma}_{j}^{\epsilon}$ are reduced, for small $\epsilon \neq 0$, we have $\widetilde{\Gamma}^{\epsilon}=\bigcup \bar{\Gamma}_{j}^{\epsilon}$. Moreover, the second property of a special deformation implies that formula (2) can be applied to each intersection $Z\left(\phi^{\epsilon}, \ldots, \phi_{n-1}^{\epsilon}\right)$. Then, from the two definitions of multiplicity of deformation, we have

$$
\#(\widetilde{\phi}, \widetilde{I})=\sum_{j} \mu_{j} \curvearrowleft\left(\widetilde{\phi}, \bar{I}_{j}\right)
$$

We are going now to extend the definition of the multiplicity $\#(\widetilde{\phi}, \widetilde{I})$ to meromorphic functions. For that we will define the multiplicities on each component $\bar{\Gamma}_{j}$ and then use formula (4) to define the multiplicity on $\widetilde{\Gamma}$.

For a meromorphic function $\widetilde{\tau}(x, \epsilon)=\widetilde{\varphi}(x, \epsilon) / \widetilde{\eta}(x, \epsilon)$ on $\bar{\Gamma}_{j}$, we set as above $\downarrow\left(\widetilde{\tau}, \bar{I}_{j}\right)=$ $\natural\left(\widetilde{\varphi}, \bar{I}_{j}\right)-\downarrow\left(\widetilde{\eta}, \bar{I}_{j}\right)$. We have seen that these multiplicities exist although $\widetilde{\varphi}$ and $\widetilde{\eta}$ are defined only on $\bar{\Gamma}_{j}$.

If $\alpha$ is a 2 -form, the restriction of $\alpha$ on the set of smooth points of $\bar{\Gamma}_{j}$ extends in a unique way to a meromorphic form on $\bar{\Gamma}_{j}$ that we denote by $\left.\alpha\right|_{\bar{\Gamma}_{j}}$ (this because $\bar{\Gamma}_{j}$ is irreducible). If $\alpha$ and $\beta$ are two 2-forms, the ratio $\left.\alpha\right|_{\bar{\Gamma}_{j}} /\left.\beta\right|_{\bar{\Gamma}_{j}}$ defines then unequivocally a meromorphic function on $\bar{\Gamma}_{j}$. Thus the multiplicities $\natural\left(\left.\alpha\right|_{\bar{\Gamma}_{j}} /\left.\beta\right|_{\bar{\Gamma}_{j}}, \bar{I}_{j}\right)$ can be defined as above. Following formula (4), we denote the sum of these multiplicities by

$$
\#\left(\frac{\left.\alpha\right|_{\widetilde{\Gamma}}}{\left.\beta\right|_{\widetilde{\Gamma}}}, \widetilde{I}\right)=\sum_{j} \mu_{j} \downarrow\left(\frac{\left.\alpha\right|_{\bar{\Gamma}_{j}}}{\left.\beta\right|_{\bar{\Gamma}_{j}}}, \bar{I}_{j}\right) .
$$

Remark 4. From now on all deformations we consider are special. Then we will use only the notation \# for multiplicities of deformation. 
Finally, we will link the multiplicity of a ratio of 1 -forms and the multiplicity of deformation of a ratio of 2 -forms.

Proposition 1. Let $I=\left(\phi_{1}, \ldots, \phi_{n-1}\right)$ be an ideal defining a 1-dimensional analytic subspace $\Gamma$ in $\mathbf{C}^{n}$. Let $\phi$ and $\psi$ be two analytic functions such that the multiplicity $e\left(\frac{\left.d \phi\right|_{\Gamma}}{\left.d \psi\right|_{\Gamma}}, I\right)$ is defined. Let $\widetilde{\phi}, \widetilde{\psi}$ and $\widetilde{I}$ be deformations of $\phi, \psi$ and $I$. Then we have

$$
e\left(\frac{\left.d \phi\right|_{\Gamma}}{\left.d \psi\right|_{\Gamma}}, I\right)=\#\left(\frac{\left.(d \widetilde{\phi} \wedge d \epsilon)\right|_{\widetilde{\Gamma}}}{\left.(d \widetilde{\psi} \wedge d \epsilon)\right|_{\widetilde{\Gamma}}}, \widetilde{I}\right) .
$$

(Recall that the two sides of this equality are defined respectively by (3) and (5).)

Proof. We use the notation introduced above, i.e. $\Gamma_{i}$ (resp. $\bar{\Gamma}_{j}$ ) is an irreducible component of $\Gamma$ (resp. $\widetilde{\Gamma}$ ) with a multiplicity $\nu_{i}$ (resp. $\mu_{j}$ ). Moreover, if $\bar{\Gamma}_{j}^{0}$ is the intersection $\bar{\Gamma}_{j} \cap\{\epsilon=0\}$, it is included in $\Gamma$ (as a set). Therefore its irreducible components are $\Gamma_{i}$ with multiplicities $\rho_{i, j}$ (maybe zero). The multiplicities $\nu_{i}, \mu_{j}$ and $\rho_{i, j}$ are related by (see [11])

$$
\sum_{j} \mu_{j} \rho_{i, j}=\nu_{i}
$$

On each component $\bar{\Gamma}_{j}$ of $\bar{\Gamma}$ we consider the meromorphic function

$$
\widetilde{\tau}_{j}=\frac{\left.(d \widetilde{\phi} \wedge d \epsilon)\right|_{\bar{\Gamma}_{j}}}{\left.(d \widetilde{\psi} \wedge d \epsilon)\right|_{\bar{\Gamma}_{j}}} .
$$

For each irreducible component $\Gamma_{i}$ of $\bar{\Gamma}_{j}^{0}$, it can be shown (with the same arguments as in Lemma 5 below) that

$$
\left.\widetilde{\tau_{j}}\right|_{\Gamma_{i}}=\frac{\left.d \phi\right|_{\Gamma_{i}}}{\left.d \psi\right|_{\Gamma_{i}}}
$$

Since we have assumed that $e\left(\frac{\left.d \phi\right|_{\Gamma}}{\left.d \psi\right|_{\Gamma}}, I\right)$ is defined, the zeroes of $\left.\widetilde{\tau_{j}}\right|_{\Gamma_{i}}$ are isolated. If we write $\widetilde{\tau}_{j}=\widetilde{\varphi} / \widetilde{\eta}$, then the zeroes of $\left.\widetilde{\varphi}\right|_{\Gamma_{i}}$ and $\left.\widetilde{\eta}\right|_{\Gamma_{i}}$ are isolated. Using Remark 3, this implies $\#\left(\widetilde{\varphi}, \bar{I}_{j}\right)=e\left(\varphi, \bar{I}_{j}^{0}\right)$ and $\#\left(\widetilde{\eta}, \bar{I}_{j}\right)=e\left(\eta, \bar{I}_{j}^{0}\right)$, where $\varphi($ resp. $\eta)$ denotes the restriction of $\widetilde{\varphi}$ (resp. $\widetilde{\eta}$ ) to $\{\epsilon=0\}$ and $\bar{I}_{j}^{0}$ the ideal associated to $\bar{\Gamma}_{j}^{0}$. From formula (2) we have then

$$
\#\left(\widetilde{\varphi}, \bar{I}_{j}\right)=\sum_{i} \rho_{i, j} e\left(\varphi, I_{i}\right), \#\left(\widetilde{\eta}, \bar{I}_{j}\right)=\sum_{i} \rho_{i, j} e\left(\eta, I_{i}\right) .
$$

But, by definition, $\#\left(\widetilde{\tau}_{j}, \bar{I}_{j}\right)=\#\left(\widetilde{\varphi}, \bar{I}_{j}\right)-\#\left(\widetilde{\eta}, \bar{I}_{j}\right)$, so we have

$$
\#\left(\widetilde{\tau}_{j}, \bar{I}_{j}\right)=\sum_{i} \rho_{i, j} e\left(\frac{\varphi}{\eta}, I_{i}\right)
$$

Since $\varphi / \eta$ restricted to $\Gamma_{i}$ is equal to $\left.\widetilde{\tau_{j}}\right|_{\Gamma_{i}}$, formula (7) gives

$$
\#\left(\widetilde{\tau}_{j}, \bar{I}_{j}\right)=\sum_{i} \rho_{i, j} e\left(\frac{\left.d \phi\right|_{\Gamma_{i}}}{\left.d \psi\right|_{\Gamma_{i}}}, I_{i}\right) .
$$

By using definitions (5) and (3) with formula (6), we obtain finally

$$
\#\left(\frac{\left.(d \widetilde{\phi} \wedge d \epsilon)\right|_{\widetilde{\Gamma}}}{\left.(d \widetilde{\psi} \wedge d \epsilon)\right|_{\tilde{\Gamma}}}, \widetilde{I}\right)=\sum_{i, j} \mu_{j} \rho_{i, j} e\left(\frac{\left.d \phi\right|_{\Gamma_{i}}}{\left.d \psi\right|_{\Gamma_{i}}}, I_{i}\right)=e\left(\frac{\left.d \phi\right|_{\Gamma}}{\left.d \psi\right|_{\Gamma}}, I\right)
$$


2.3. Result for isolated intersections. We will now establish a preliminary result that will be useful in the next section. This result is in fact a particular case of a theorem proved by Gabrielov (see [6], Theorem 1.1). We give here a simplified proof for our case.

Lemma 2. Let $I=\left(\phi_{1}, \ldots, \phi_{n-1}\right)$ be an ideal such that $\Gamma$ defined as $\left\{\phi_{1}=\ldots\right.$ $\left.\ldots=\phi_{n-1}=0\right\}$ is a 1-dimensional analytic subspace in $\mathbf{C}^{n}$. Let $\phi$ and $\psi$ be analytic functions such that $Z(\phi, I)$ and $Z(\psi, I)$ are isolated intersections. Then we have

$$
e(\phi, I)=e\left(\psi \frac{\left.d \phi\right|_{\Gamma}}{\left.d \psi\right|_{\Gamma}}, I\right) .
$$

P r o of. We first choose a system of coordinates $\left(x_{1}, \ldots, x_{n}\right)$ in a neighborhood of the origin. Let $\Gamma=\bigcup \Gamma_{j}$ be the decomposition of $\Gamma$ into irreducible components.

Since $Z(\psi, I)$ is isolated, for each component $\Gamma_{j}$ there is an analytic parametrization (Puiseux) such that

where $x_{i}(t)$ are analytic in $t$.

$$
\left\{\begin{array}{l}
\left.\psi\right|_{\Gamma_{j}}=t^{N_{j}} \\
\left.x_{i}\right|_{\Gamma_{j}}=x_{i}(t) \quad i=1, \ldots, n
\end{array}\right.
$$

The function $\left.\phi\right|_{\Gamma_{j}}$ does not vanish identically since $Z(\phi, I)$ is isolated. Hence $\left.\phi\right|_{\Gamma_{j}}$ has a Puiseux expansion at the origin:

$$
\left.\phi\right|_{\Gamma_{j}}=c_{j} t^{r_{j}}+o\left(t^{r_{j}}\right)=c_{j} \psi^{\frac{r_{j}}{N_{j}}}+o\left(\psi^{\frac{r_{j}}{N_{j}}}\right)
$$

where $r_{j}$ is a positive integer and $c_{j} \neq 0$ a constant. If $I_{j}$ is the ideal associated to $\Gamma_{j}$, the order of vanishing $r_{j}$ of $\left.\phi\right|_{\Gamma_{j}}$ is equal to $e\left(\phi, I_{j}\right)$ ([3], p. 8). From formula $(2)$, we have $e(\phi, I)=\sum_{j} \nu_{j} r_{j}$.

Let us now differentiate the relation $(8)$

$$
\left.d \phi\right|_{\Gamma_{j}}=\left.\left(c_{j} \frac{r_{j}}{N_{j}} \psi^{\frac{r_{j}}{N_{j}}-1}+o\left(\psi^{\frac{r_{j}}{N_{j}}-1}\right)\right) d \psi\right|_{\Gamma_{j}}
$$

We then have a Puiseux expansion for the function $\psi \frac{\left.d \phi\right|_{\Gamma_{j}}}{\left.d \psi\right|_{\Gamma_{j}}}$ :

$$
\psi \frac{\left.d \phi\right|_{\Gamma_{j}}}{\left.d \psi\right|_{\Gamma_{j}}}=c_{j} \frac{r_{j}}{N_{j}} \psi^{\frac{r_{j}}{N_{j}}}+o\left(\psi^{\frac{r_{j}}{N_{j}}}\right)=c_{j} \frac{r_{j}}{N_{j}} t^{r_{j}}+o\left(t^{r_{j}}\right) .
$$

Hence the functions $\left.\phi\right|_{\Gamma_{j}}$ and $\psi \frac{\left.d \phi\right|_{\Gamma_{j}}}{\left.d \psi\right|_{\Gamma_{j}}}$ have expansions of the same order, that is $e\left(\phi, I_{j}\right)=$ $e\left(\psi \frac{\left.d \phi\right|_{\Gamma_{j}}}{\left.d \psi\right|_{\Gamma_{j}}}, I_{j}\right)$. From formula (2) and definition (3), we have

$$
e\left(\psi \frac{\left.d \phi\right|_{\Gamma}}{\left.d \psi\right|_{\Gamma}}, I\right)=e(\phi, I)
$$

\section{Multiplicity of a polynomial on the trajectory of a polynomial vector} field

THEOREM 3. Let $\xi(x)=\sum_{i=1}^{3} X_{i}(x) \frac{\partial}{\partial x_{i}}$ be a vector field on $\mathbf{C}^{3}$, with polynomial in $x$ coefficients $X_{i}$ of degree not exceeding $d$ and such that $\xi(O) \neq 0$. Let $\gamma$ be the trajectory of $\xi$ through the origin.

Then, if $P(x)$ is a polynomial of degree $p$ such that $\left.P\right|_{\gamma} \not \equiv 0$, the order $\mu$ of $\left.P\right|_{\gamma}$ at the origin satisfies

$$
\mu \leq p+2 p(p+d-1)^{2}
$$


ExAmple. Set $\xi(x)=\frac{\partial}{\partial x_{1}}+x_{1}^{d} \frac{\partial}{\partial x_{2}}+x_{2}^{d} \frac{\partial}{\partial x_{3}}$ and $P(x)=x_{3}^{p}$. Then it can be easily seen that, in this case, $\mu=p\left(1+d+d^{2}\right)$. This proves that the estimation (9) is asymptotically optimal in terms of $p$ and $d$ when $p \leq d$.

Rem ark. Remind that in the case $n=2$, there is a bound of the form $p(p+d-1)+1$ (cf. $[4,10])$.

Proof of Theorem 3. Let $P(x)$ be a polynomial of degree $p$ with $\left.P\right|_{\gamma} \not \equiv 0$. We suppose $P(O)=0$ (if $P(O) \neq 0$, the order $\mu$ is zero). Let us remark that the estimate (9) for $P_{1} P_{2}$ follows from the estimates for $P_{1}$ and $P_{2}$. Then we may assume that $P$ is an irreducible polynomial.

The order of $\left.P\right|_{\gamma}$ at the origin is also the multiplicity of the isolated intersection $\gamma \cap\{P=0\}$. So we can use Lemma 2 to estimate $\mu$. For that we need coordinates where $\gamma$ has a simple expression.

Proposition 4. Let $l: \mathbf{C}^{3} \rightarrow \mathbf{C}$ be a linear form such that the plane $\Sigma=\{l(x)=0\}$ is transverse to the vector $\xi(O)$ and $A: \Sigma \rightarrow \mathbf{C}^{2}$ be an isomorphism. There exists an analytic change of coordinates $y=\Phi(x)$ near the origin such that, if $y=\left(y_{1}, y_{2}, z\right)$, we have:

- $\xi(y)=\frac{\partial}{\partial z}$

- $z=l(x) u(x)$, where $u$ is an analytic function with $u(O) \neq 0$;

- $\left(\left.y_{1}\right|_{l(x)=0},\left.y_{2}\right|_{l(x)=0}\right)=A(x)$ is an isomorphism.

P r o of. Let us denote by $\varphi_{t}(x)$ the flow of $\xi(x)$. We consider the map $\Psi$ defined in a neighborhood of the origin by

$$
\Psi\left(y_{1}, y_{2}, z\right)=\varphi_{z}\left(A^{-1}\left(y_{1}, y_{2}\right)\right) .
$$

It can be shown that the matrix of the mapping $T_{O} \Psi$ is $\left(A^{-1} \mid \xi(O)\right)\left(A^{-1}\right.$ is a $(3 \times 2)$ matrix and $\xi(O)$ a column vector). The map $T_{O} \Psi$ is an isomorphism because $\Sigma$, the plane $\{l(x)=0\}$, is transverse to $\xi(O)$ and $A^{-1}$ is an isomorphism from $\mathbf{C}^{2}$ to $\Sigma$. The rest of the proof is classical in differential geometry: near the origin $\Psi$ is a diffeomorphism and by setting $\Phi=\Psi^{-1}$ we have the properties required in the proposition.

In the new coordinates $y$ the trajectory $\gamma$ is given by the equations $\left\{y_{1}=y_{2}=0\right\}$ and we have to estimate the multiplicity $\mu=e\left(P(y), y_{1}, y_{2}\right)$. We want to apply Lemma 2 to the ideal $I=\left(P(y), y_{2}\right)$ (which defines a curve $\Gamma$ ) and the functions $\phi(y)=y_{1}$ and $\psi(y)=z$. We can always choose the isomorphism $A$ of Proposition 4 in such a way that the hypothesis of the lemma are guaranteed. We have then

$$
\mu=e(\phi, I)=e\left(\psi \frac{\left.d \phi\right|_{\Gamma}}{\left.d \psi\right|_{\Gamma}}, I\right)=e\left(z \frac{\left.d y_{1}\right|_{\Gamma}}{\left.d z\right|_{\Gamma}}, I\right) .
$$

LEMMA 5. Let $\left(y_{1}, \ldots, y_{n}\right)$ be coordinates on $\mathbf{C}^{n}$, and $\Upsilon$ be an irreducible component of the $(n-2)$-dimensional subspace of $\mathbf{C}^{n}$ defined by $\{\alpha=\beta=0\}$, where the functions $\alpha$ and $\beta$ are analytic. Let $\omega$ and $\omega^{\prime}$ be analytic $(n-2)$-forms over $\mathbf{C}^{n}$ such that, for all $y \in \Upsilon,\left(\left.\omega^{\prime}\right|_{\Upsilon}\right)_{y} \neq 0$.

Then there is an analytic function $f$ such that, for $y \in \Upsilon$, we have

$$
f(y) \frac{\left(\left.\omega\right|_{\Upsilon}\right)_{y}}{\left(\left.\omega^{\prime}\right|_{\Upsilon}\right)_{y}}=\frac{(\omega \wedge d \alpha \wedge d \beta)_{y}}{\left(d y_{1} \wedge \ldots \wedge d y_{n}\right)_{y}} .
$$


Proof. For $y \in \Upsilon$, we set $r(y)=\frac{\left(\left.\omega\right|_{\Upsilon}\right)_{y}}{\left(\omega^{\prime} \mid \Upsilon\right)_{y}}$ (this ratio is defined since $\left.\left(\left.\omega^{\prime}\right|_{\Upsilon}\right)_{y} \neq 0\right)$. Let $y$ be a smooth point of $\Upsilon$ and $\left(v_{1}, \ldots, v_{n-2}\right)$ be a basis of $T_{y} \Upsilon$. The restricted forms are given by $\left(\left.\omega\right|_{\Upsilon}\right)_{y}\left(v_{1}, \ldots, v_{n-2}\right)=\omega_{y}\left(v_{1}, \ldots, v_{n-2}\right)$ and in the same way $\left(\left.\omega^{\prime}\right|_{\Upsilon}\right)_{y}\left(v_{1}, \ldots, v_{n-2}\right)=$ $\omega_{y}^{\prime}\left(v_{1}, \ldots, v_{n-2}\right) \neq 0$. Thus we have $r(y)=\frac{\omega_{y}\left(v_{1}, \ldots, v_{n-2}\right)}{\omega_{y}^{\prime}\left(v_{1}, \ldots, v_{n-2}\right)}$.

Let us complete $v_{1}, \ldots, v_{n-2}$ in a basis $\left(v_{1}, \ldots, v_{n}\right)$ of $\mathbf{C}^{n}$. We want to evaluate the $n$-forms $(\omega \wedge d \alpha \wedge d \beta)_{y}$ and $\left(\omega^{\prime} \wedge d \alpha \wedge d \beta\right)_{y}$ on this basis. Let us recall that, since $\Upsilon$ is a component of the subspace $\{\alpha=\beta=0\}$, we have $d \alpha_{y}\left(v_{i}\right)=d \beta_{y}\left(v_{i}\right)=0$ for $i=1, \ldots, n-2$ and then:

$$
\begin{aligned}
(\omega \wedge d \alpha \wedge d \beta)_{y}\left(v_{1}, \ldots, v_{n}\right) & =\omega_{y}\left(v_{1}, \ldots, v_{n-2}\right)(d \alpha \wedge d \beta)_{y}\left(v_{n-1}, v_{n}\right) \\
\left(\omega^{\prime} \wedge d \alpha \wedge d \beta\right)_{y}\left(v_{1}, \ldots, v_{n}\right) & =\omega_{y}^{\prime}\left(v_{1}, \ldots, v_{n-2}\right)(d \alpha \wedge d \beta)_{y}\left(v_{n-1}, v_{n}\right) .
\end{aligned}
$$

From the other hand, since $\alpha, \beta$ and the form $\omega^{\prime}$ are analytic in $y=\left(y_{1}, \ldots, y_{n}\right)$, we have $\left(\omega^{\prime} \wedge d \alpha \wedge d \beta\right)_{y}=f(y)\left(d y_{1} \wedge \ldots \wedge d y_{n}\right)_{y}$, with $f$ analytic in $y=\left(y_{1}, \ldots, y_{n}\right)$. Since $\omega_{y}^{\prime}\left(v_{1}, \ldots, v_{n-2}\right) \neq 0$ it is clear from (12) that $f(y)=0$ if and only if $(d \alpha \wedge d \beta)_{y}=0$.

Consider now the ratio $r^{\prime}(y)=\frac{(\omega \wedge d \alpha \wedge d \beta)_{y}}{\left(d y_{1} \wedge \ldots \wedge d y_{n}\right)_{y}}$. If $(d \alpha \wedge d \beta)_{y} \neq 0$ (and then $\left.f(y) \neq 0\right)$, $r^{\prime}(y)$ is equal to $f(y) r(y)$ (this follows from (11), (12) and the expression of $\left.\left(\omega^{\prime} \wedge d \alpha \wedge d \beta\right)_{y}\right)$.

If $(d \alpha \wedge d \beta)_{y}=0$, then $r^{\prime}(y)=0$. But we have also $f(y)=0$ and then $f(y) r(y)=0$. Thus in both cases we have $r^{\prime}(y)=f(y) r(y)$.

Let us apply this lemma to the 1-forms $\omega=z d y_{1}, \omega^{\prime}=d z$ and to an irreducible component $\Gamma_{j}$ of $\Gamma$. The property $\left.d z\right|_{\Gamma_{j}} \neq 0$ is satisfied since $Z\left(z, P, y_{2}\right)$ is an isolated intersection. We obtain

$$
f(y) \frac{\left.z d y_{1}\right|_{\Gamma_{j}}}{\left.d z\right|_{\Gamma_{j}}}=\frac{\left(z d y_{1} \wedge d P \wedge d y_{2}\right)_{y}}{\left(d y_{1} \wedge d y_{2} \wedge d z\right)_{y}}=z \frac{\partial P}{\partial z} .
$$

Reminding notations of Subsection 2.2, we have

$$
e\left(\frac{\left.z d y_{1}\right|_{\Gamma_{j}}}{\left.d z\right|_{\Gamma_{j}}}, I_{j}\right)=e\left(z \frac{\partial P}{\partial z}, I_{j}\right)-e\left(f, I_{j}\right) \leq e\left(z \frac{\partial P}{\partial z}, I_{j}\right) .
$$

Finally, it follows from formulae (3) and (10) that the multiplicity $\mu$ satisfies

$$
\mu \leq e\left(z \frac{\partial P}{\partial z}, I\right) \leq \mu_{1}+\mu_{2}
$$

where $\mu_{1}=e(z, I)$ and $\mu_{2}=e\left(\frac{\partial P}{\partial z}, I\right)$.

We first calculate $\mu_{1}=e\left(z, P(y), y_{2}\right)$. In the original coordinates $x$ we can write $\mu_{1}$ as $\mu_{1}=e\left(P(x), l(x), y_{2}\right)=e\left(\left.P(x)\right|_{l(x)=0},\left.y_{2}\right|_{l(x)=0}\right)$ (see Proposition 4). The intersection is isolated (remind that it was required to write formula (10)) and all the terms are polynomials in $x$. The estimate follows from Bézout's theorem:

$$
\mu_{1} \leq p
$$

The second term $\mu_{2}$ is $e\left(\frac{\partial P}{\partial z}(y), P(y), y_{2}\right)$. Notice that $\frac{\partial P}{\partial z}$ cannot be divisible by $P$. As $P$ is irreducible, we can choose the mapping $l$ and $A$ of Proposition 4 such that the intersections $Z\left(\frac{\partial P}{\partial z}(y), P(y), y_{2}\right)$ and $Z\left(\frac{\partial P}{\partial z}(y), P(y), z\right)$ are isolated. We can then apply again Lemma 2 to the ideal $I^{\prime}=\left(P(y), \frac{\partial P}{\partial z}(y)\right)$ (which defines a curve $\Gamma^{\prime}$ ) and to the functions $\phi^{\prime}(y)=y_{2}$ and $\psi(y)=z$, and obtain

$$
\mu_{2}=e\left(\phi^{\prime}, I^{\prime}\right)=e\left(\psi \frac{\left.d \phi^{\prime}\right|_{\Gamma^{\prime}}}{\left.d \psi\right|_{\Gamma^{\prime}}}, I^{\prime}\right)
$$


Therefore we can write $\mu_{2}=\mu_{3}+\mu_{4}$ with $\mu_{3}=e\left(z, I^{\prime}\right)$ and $\mu_{4}=e\left(\frac{\left.d y_{2}\right|_{\Gamma^{\prime}}}{\left.d z\right|_{\Gamma^{\prime}}}, I^{\prime}\right)$ (this last multiplicity is defined since $Z\left(\frac{\partial P}{\partial z}(y), P(y), z\right)$ is isolated and $\mu_{2}$ is defined).

We first estimate $\mu_{3}=e\left(z, P(y), \frac{\partial P}{\partial z}(y)\right)$. Recall that $\frac{\partial P}{\partial z}$ is the Lie derivation $\xi . P$ of $P$ by $\xi$. In the original coordinates, this derivation is given by $\xi \cdot P(x)=\sum_{i=1}^{3} X_{i}(x) \frac{\partial P}{\partial x_{i}}(x)$. Thus this is a polynomial in $x$ of degree not exceeding $p+d-1 ; \mu_{3}=e(l(x), P(x), \xi . P(x))$ is then the multiplicity of a polynomial intersection. From Bézout's theorem we have

$$
\mu_{3} \leq p(p+d-1)
$$

Let us introduce now a deformation (as defined in Subsection 2.2) of $P$ given in the original coordinates by: $\widetilde{P}(x, \epsilon)=P(x)-\epsilon(a+b l(x))$ where $a, b \in \mathbf{C}$ are generic. $P^{\epsilon}$ is polynomial in $x$ of degree $p$. In the new coordinates this is a deformation $\widetilde{P}(y, \epsilon)$ of $P(y)$.

We will express $\mu_{4}$ by using the multiplicities of deformations (see Subsection 2.2). Since the multiplicity $\mu_{4}$ is defined, we have from Proposition 1

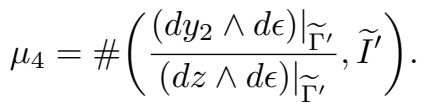

We can apply now Lemma 5 to the 2-forms $\left(d y_{2} \wedge d \epsilon\right)$ and $(d z \wedge d \epsilon)$ and to the irreducible components of $\widetilde{\Gamma}^{\prime}$. We have seen that $Z\left(\frac{\partial P}{\partial z}(y), P(y), z\right)$ is an isolated intersection, so the condition $\left.(d z \wedge d \epsilon)\right|_{\widetilde{\Gamma}^{\prime}} \neq 0$ is satisfied.

By using formula (4) and the argument of formula (13), we have, assuming that $\mu_{4}^{\prime}$ is the multiplicity of a special deformation (recall that formula (4) is true only for special deformations)

$$
\mu_{4}=\#\left(\frac{\left.\left(d y_{2} \wedge d \epsilon\right)\right|_{\widetilde{\Gamma}^{\prime}}}{(d z \wedge d \epsilon) \widetilde{\Gamma}_{\widetilde{\Gamma}^{\prime}}}\right) \leq \#\left(\frac{d\left(\frac{\partial \widetilde{P}}{\partial z}\right) \wedge d \widetilde{P} \wedge d y_{2} \wedge d \epsilon}{d y_{1} \wedge d y_{2} \wedge d z \wedge d \epsilon}, \widetilde{I}^{\prime}\right)=\mu_{4}^{\prime} .
$$

We have:

$$
\frac{d\left(\frac{\partial \widetilde{P}}{\partial z}\right) \wedge d \widetilde{P} \wedge d y_{2} \wedge d \epsilon}{d y_{1} \wedge d y_{2} \wedge d z \wedge d \epsilon}=\left|\begin{array}{cccc}
\frac{\partial^{2} \widetilde{P}}{\partial z \partial y_{1}} & \frac{\partial \widetilde{P}}{\partial y_{1}} & 0 & 0 \\
\frac{\partial^{2} \widetilde{P}}{\partial z \partial y_{2}} & \frac{\partial \widetilde{P}}{\partial y_{2}} & 1 & 0 \\
\frac{\partial^{2} \widetilde{P}}{\partial z^{2}} & \frac{\partial \widetilde{P}}{\partial z} & 0 & 0 \\
\frac{\partial^{2} \widetilde{P}}{\partial z \partial \epsilon} & \frac{\partial \widetilde{P}}{\partial \epsilon} & 0 & 1
\end{array}\right|=\frac{\partial \widetilde{P}}{\partial y_{1}} \frac{\partial^{2} \widetilde{P}}{\partial z^{2}}-\frac{\partial^{2} \widetilde{P}}{\partial z \partial y_{1}} \frac{\partial \widetilde{P}}{\partial z} .
$$

Since $\frac{\partial \widetilde{P}}{\partial z}(y)=0$ for $y \in \widetilde{\Gamma}^{\prime}$, we can write $\mu_{4}^{\prime}$ as

$$
\mu_{4}^{\prime}=\#\left(\frac{\partial \widetilde{P}}{\partial y_{1}} \frac{\partial^{2} \widetilde{P}}{\partial z^{2}}, \widetilde{I}^{\prime}\right)
$$

Thus $\mu_{4}^{\prime}$ will be the multiplicity of a special deformation if both deformations $\left(\frac{\partial^{2} \widetilde{P}}{\partial z^{2}}, \widetilde{I^{\prime}}\right)$ and $\left(\frac{\partial \widetilde{P}}{\partial y_{1}}, \widetilde{I}^{\prime}\right)$ are special. We will have then $\mu_{4}^{\prime}=\mu_{5}+\mu_{6}$ with $\mu_{5}=\#\left(\frac{\partial^{2} \widetilde{P}}{\partial z^{2}}, \widetilde{I}^{\prime}\right)$ and $\mu_{6}=\#\left(\frac{\partial \widetilde{P}}{\partial y_{1}}, \widetilde{I}^{\prime}\right)$.

Proposition 6. Let $Q(x)=P(x)-a-b l(x)$. Then, for generic $a$ and $b$, the intersection $\left\{Q=\frac{\partial Q}{\partial z}=\frac{\partial^{2} Q}{\partial z^{2}}=0\right\}$ is isolated.

Proof. Let us first notice that, from Proposition 4, we have $z=l(x) u(x)$, with $u(O) \neq 0$. Therefore $\frac{\partial l}{\partial z}$ is invertible near the origin. Since $\frac{\partial Q}{\partial z}=\frac{\partial Q}{\partial l} \frac{\partial l}{\partial z}$, the functions $\frac{\partial Q}{\partial z}$ 
and $\frac{\partial Q}{\partial l}$ have the same zeroes. Moreover,

$$
\frac{\partial^{2} Q}{\partial z^{2}}=\frac{\partial^{2} Q}{\partial l^{2}}\left(\frac{\partial l}{\partial z}\right)^{2}+\frac{\partial Q}{\partial l} \frac{\partial^{2} l}{\partial z^{2}},
$$

so $\left\{Q=\frac{\partial Q}{\partial z}=\frac{\partial^{2} Q}{\partial z^{2}}=0\right\}$ has the same zeroes as $\left\{Q=\frac{\partial Q}{\partial l}=\frac{\partial^{2} Q}{\partial l^{2}}=0\right\}$. Then we have to prove that, for generic $a$ and $b$, the intersection $\left\{P-a-b l=\frac{\partial P}{\partial l}-b=\frac{\partial^{2} P}{\partial l^{2}}=0\right\}$ is isolated.

We can always assume $P(O)=\frac{\partial P}{\partial z}(O)=0$ (otherwise $\mu \leq 2$ ), and then $\frac{\partial P}{\partial l}(O)=0$. Since $P$ is not identically zero, this implies that $\frac{\partial^{2} P}{\partial l^{2}}$ is not identically zero either. Thus $\left\{\frac{\partial^{2} P}{\partial l^{2}}=0\right\}$ has codimension one. Locally this subspace has finitely many connected components. For a generic $b$, the function $\frac{\partial P}{\partial l}$ does not have a constant value $b$ on any of these components of codimension 1. Hence, for a generic $b$, the subspace $\left\{\frac{\partial^{2} P}{\partial l^{2}}=\frac{\partial P}{\partial l}-b=0\right\}$ has codimension at least two. Let us fix such a generic $b$.

We can repeat the same arguments with $\left\{\frac{\partial^{2} P}{\partial l^{2}}=\frac{\partial P}{\partial l}-b=0\right\}$ instead of $\left\{\frac{\partial^{2} P}{\partial l^{2}}=0\right\}$ and $P-b l=a$ instead of $\frac{\partial P}{\partial l}=b$. This shows that $\left\{\frac{\partial^{2} P}{\partial l^{2}}=\frac{\partial P}{\partial l}-b=P-b l-a=0\right\}$ has codimension at least 3 , for a generic $a$. That means that the intersection $\left\{\frac{\partial^{2} Q}{\partial l^{2}}=\frac{\partial Q}{\partial l}=\right.$ $Q=0\}$ is isolated and the proof is done.

This proposition, applied to $Q(x)=P^{\epsilon}(x)$, for small $\epsilon \neq 0$, implies that the deformation $\left(\frac{\partial^{2} \widetilde{P}}{\partial z^{2}}, \widetilde{I}^{\prime}\right)$ is special. Furthermore, $\mu_{5}$ can be estimated in the same way as $\mu_{3}$ : in the original coordinates $\mu_{5}=\#\left(\widetilde{P}(x, \epsilon), \xi \cdot \widetilde{P}(x, \epsilon), \xi^{2} \cdot \widetilde{P}(x, \epsilon)\right)$ where $\widetilde{P}, \xi \cdot \widetilde{P}$ and $\xi^{2} \cdot \widetilde{P}$ are polynomials in $x$ of degree not greater than, respectively, $p, p+d-1$ and $p+2(d-1)$. We apply Remark 2 and obtain

$$
\mu_{5} \leq p(p+d-1)(p+2(d-1)) .
$$

The same kind of arguments as in the proof of Proposition 6 proves that if $Q(y)$ is an analytic function such that $\frac{\partial Q}{\partial z}$ is not identically zero, then, for a generic $a$, the intersection $\left\{Q-a=\frac{\partial Q}{\partial z}=\frac{\partial Q}{\partial y_{1}}=0\right\}$ is isolated. By setting $Q(y)=P(y)-b l(y) \epsilon$, for small $\epsilon \neq 0$, it follows that $\left(\widetilde{P}, \frac{\partial \widetilde{P}}{\partial z}, \frac{\partial \widetilde{P}}{\partial y_{1}}\right)$ is a special deformation. To estimate the multiplicity $\mu_{6}$, we would like to replace $\frac{\partial \widetilde{P}}{\partial y_{1}}$ by a polynomial in $x$ and then apply Bézout's theorem. This will be possible, due to the following lemma.

Lemma 7. There exists a vector field $\xi_{0}(x)=\xi_{0}$ constant in the coordinates $x$ such that

$$
\#\left(\widetilde{P}, \frac{\partial \widetilde{P}}{\partial z}, \frac{\partial \widetilde{P}}{\partial y_{1}}\right)=\#\left(\widetilde{P}, \frac{\partial \widetilde{P}}{\partial z}, \xi_{0} \cdot \widetilde{P}\right)
$$

This lemma allows to write $\mu_{6}=\#\left(\widetilde{P}(x, \epsilon), \xi \cdot \widetilde{P}(x, \epsilon), \xi_{0} \cdot \widetilde{P}(x, \epsilon)\right)$. By applying Remark 2 we have then

$$
\mu_{6} \leq p(p+d-1)(p-1) .
$$

Finally, the multiplicity $\mu$ satisfies $\mu \leq \mu_{1}+\mu_{3}+\mu_{5}+\mu_{6}$. By using estimates (14), (16), (17), (18) we have

$$
\mu \leq p+2 p(p+d-1)^{2}
$$

which is the estimate required in Theorem 3 . 
Proof of Lemma 7 . Let us recall that $\#\left(\widetilde{P}, \frac{\partial \widetilde{P}}{\partial z}, \frac{\partial \widetilde{P}}{\partial y_{1}}\right)$ is defined as the number of isolated zeroes, counted with their multiplicities, of $\left\{P^{\epsilon}=\frac{\partial P^{\epsilon}}{\partial z}=\frac{\partial P^{\epsilon}}{\partial y_{1}}=0\right\}, \epsilon \neq 0$, converging to the origin $O$ when $\epsilon \rightarrow 0$.

Let $\gamma$ be the curve defined by the ideal $\left(P, \frac{\partial P}{\partial z}\right)(\gamma$ is a curve because we assume $P$ irreducible). If $x \in \gamma$, let $T(x)$ be the tangent to $\gamma$ at $x$, and $L(x)$ be the 2-plane (vector space) spanned by $T(x)$ and the $z$-axis $L_{z}(L(x)$ is a plane for $x$ small and $\neq O$, because by hypothesis $\gamma$ does not contain the $z$-axis which is the integral curve of $\xi$ ). If $x$ tends to 0 , the planes $L(x)$ have a finite number of limit positions $L_{i}, 1 \leq i \leq l$ (at most one for each local irreducible component of $\gamma$ ). Let $\xi_{0}$ be an analytic vector field such that $\xi_{0}(O) \notin L_{i}, 1 \leq i \leq l$, and $M(x)$ be the 2-plane spanned by $\xi_{0}(x)$ and $L_{z}(M(x)$ is a 2-plane for small $x$ because by hypothesis $\left.\xi_{0}(O) \notin L_{i}\right)$.

Let $B_{\delta}$ be the ball of center $O$ and radius $\delta, S_{\delta}=\partial B_{\delta}$.

Proposition 8. Under the above hypothesis, there exists $\delta_{0}>0$ such that for $\delta \leq \delta_{0}$, there exists $\epsilon_{0}$ such that the system

$$
P^{\epsilon}=\frac{\partial P^{\epsilon}}{\partial z}=\xi_{0} P^{\epsilon}=0
$$

has no solution on $S_{\delta}$ for $\epsilon \leq \epsilon_{0}$.

Proof. Let $d$ be a distance function on the projective space of 2-planes in $\mathbf{C}^{3}$. Hypothesis $\xi_{0}(O) \notin L_{i}$ implies that there exist $C>0$ and $\delta_{0}>0$ such that for any $\delta \leq \delta_{0}$ we have $d\left(L(x), M\left(x^{\prime}\right)\right)>C$ for $x \in \gamma \cap S_{\delta}, x^{\prime} \in S_{\delta}$ (by continuity of $d$ ). If $x^{\epsilon}$ is a solution of (19), the tangent plane to the (smooth) surface $S^{\epsilon}=\left\{P^{\epsilon}=0\right\}$ is $M(x)$, and it is close to $L\left(x^{\prime}\right)$ for $x^{\prime} \in \gamma \cap S_{\delta}$ (this is because near a smooth point $x \in \gamma$, the tangent to $\gamma^{\epsilon}$ at a point $x^{\epsilon}$ is close to the tangent $T(x)$ to $\gamma$ ). But that is impossible if $\delta \leq \delta_{0}$, since $d\left(L(x), M\left(x^{\prime}\right)\right)>C$.

This proposition implies easily the following facts:

- There exists $\delta_{0}>0$ such that for $\epsilon$ small enough, the set defined in $B_{\delta_{0}}$ by the system (19) is made with isolated points. In fact, if not, the set of solutions of (19) would contain a connected component of $\gamma^{\epsilon} \cap B_{\delta}$, which is impossible, because such a component must intersect $S_{\delta}$.

- The limit (when $\epsilon \rightarrow 0$ ) of all solutions of (19) is $O$. In fact, if there exist no sequence of such limit points converging to $O$, we can choose $\delta_{0}$, small enough such that $B_{\delta}$ does not contain any limit point. If there exists a sequence of such limit points converging to $O$, for any $\delta_{0}$, we would find a solution of (19) in $S_{\delta}$ for some $\delta \leq \delta_{0}$ for arbitrarily small $\epsilon$.

These two facts prove that the number of solutions of (19) in $B_{\delta}$ (for small $\delta$ ), is exactly the number $\#\left(\widetilde{P}, \frac{\partial \widetilde{P}}{\partial z}, \xi_{0} . \widetilde{P}\right)$ we are looking for.

Now, set $\xi_{1}=\partial / \partial y_{1}$. In the $x$-coordinates, set $\xi_{1}=\xi_{1}(O)+\xi_{2}, \xi_{t}=\xi_{1}(O)+t \xi_{2}$. Then, by compactness of $[0,1]$, we may assume that for $\delta \leq \delta_{0}$, the conclusion of Proposition 8 is valid for any $t \in[0,1]$. Then, for $\epsilon \leq \epsilon_{0}$, the number of solutions of

$$
P^{\epsilon}=\frac{\partial P^{\epsilon}}{\partial z}=\xi_{t} P^{\epsilon}=0
$$

in the ball $B_{\delta}$ is locally independent of $t([1]$, p. 76$)$, and so, by connectivity of $[0,1]$, constant for $t \in[0,1]$. That proves Lemma 7 . 
4. Degree of nonholonomy. Let $\Sigma=\left\{\chi_{1}, \ldots, \chi_{s}\right\}$ be a set of vector fields in $\mathbf{C}^{n}$. Let $\mathcal{L}^{1}=\mathcal{L}^{1}\left(\chi_{1}, \ldots, \chi_{s}\right)$ the set of all linear combinations, with complex coefficients, of the vector fields $\chi_{1}, \ldots, \chi_{s}$. We define recursively $\mathcal{L}^{i}=\mathcal{L}^{i}\left(\chi_{1}, \ldots, \chi_{s}\right)$ by setting

$$
\mathcal{L}^{i}=\mathcal{L}^{i-1}+\left[\mathcal{L}^{1}, \mathcal{L}^{i-1}\right]
$$

for $i \geq 2$. The union $\mathcal{L}=\mathcal{L}\left(\chi_{1}, \ldots, \chi_{s}\right)$ of all $\mathcal{L}^{i}$ is the Lie algebra generated by the vector fields $\chi_{i}$.

For $x \in \mathbf{C}^{n}$, let $L^{i}(x)$ (resp. $\left.L(x)\right)$ be the linear subspace generated by the values at $x$ of the vector fields in $\mathcal{L}^{i}(\Sigma)$ (resp. in $\mathcal{L}(\Sigma)$ ). The minimal $i$ such that $\operatorname{dim} L^{i}(x)=\operatorname{dim} L(x)$ is denoted by $r(x)$ and called degree of nonholonomy of $\Sigma$ at $x$. Due to the importance of this degree in nonholonomic control theory (see for instance [2]), the problem of bounding it arises in a natural way.

Let us consider vector fields $\chi_{1}, \ldots, \chi_{s}$ whose coordinates are polynomials of degree not exceeding $d$. The problem is to find a bound on the degree of nonholonomy in terms of degree $d$ and of dimension $n$. Let us state a result of Gabrielov [5]:

Let $x \in \mathbf{C}^{n}$. Under the above hypothesis, there exists a polynomial vector field $\xi$ of degree not exceeding $2^{n-3} d$ and a polynomial $P$ of degree not exceeding $2^{n-1} d$ such that:

- $\left.P\right|_{\gamma} \not \equiv 0$, where $\gamma$ denotes the trajectory of $\xi$ through $x$;

- if $\mu$ denotes the order of $\left.P\right|_{\gamma}$ at $x$, then

$$
r(x) \leq 2^{n-2}+\mu 2^{n-3} .
$$

Thus a bound on the order $\mu$ gives also a bound on the degree of nonholonomy. As a consequence of Theorem 3 we have then

THEOREM 9. Let $\Sigma=\left\{\chi_{1}, \ldots, \chi_{s}\right\}$ be a set of vector fields on $\mathbf{C}^{3}$ whose coordinates are polynomials of degree not greater than $d$; let $r(x)$ be the degree of nonholonomy of $\Sigma$ at $x \in \mathbf{C}^{3}$. Then we have the following upper bound:

$$
r(x) \leq 2+4 d+8 d(5 d-1)^{2} .
$$

\section{References}

[1] V. I. Arnol'd, S. M. Guseŭn-Zade and A. N. Varchenko, Singularities of Differentiable Maps, Birkhäuser, Boston, 1985.

[2] A. Bellaïche, The tangent space in sub-Riemannian geometry, in: Sub-Riemannian Geometry, A. Bellaïche and J.-J. Risler (ed.), Progr. Math. 144, Birkhäuser, Basel, 1996, 1-78.

[3] W. Fulton, Intersection Theory, Ergeb. Math. Grenzgeb. (3) 2, Springer, Berlin, 1984.

[4] A. Gabrielov, J.-M. Lion and R. Moussu, Ordre de contact de courbes intégrales du plan, C. R. Acad. Sci. Paris Sér. I Math. 319 (1994), 219-221.

[5] A. Gabrielov, Multiplicities of zeroes of polynomials on trajectories of polynomial vector fields and bounds on degree of nonholonomy, Math. Res. Lett. 2 (1995), 437-451.

[6] A. Gabrielov, Multiplicities of Pfaffian intersections and the Eojasiewicz inequality, Selecta Math. (N. S.) 1 (1995), 113-127.

[7] A. Gabrielov, Multiplicity of a Zero of an Analytic Function on a Trajectory of a Vector Field, Preprint, Purdue University, March 1997. 
[8] E. Kunz, Introduction to Commutative Algebra and Algebraic Geometry, Birkhäuser, Boston, 1985.

[9] Y. V. Nesterenko, Estimates for the number of zeros of certain functions, in: New Advances in Transcendence Theory, A. Baker (ed.), Cambridge Univ. Press, Cambridge, 1988, 263-269.

[10] J.-J. Risler, A bound for the degree of nonholonomy in the plane, Theoret. Comput. Sci. 157 (1996), 129-136.

[11] P. Samuel, Méthodes d'algèbre abstraite en géométrie algébrique, Ergeb. Math. Grenzgeb. 4, Springer, Berlin, 1967. 